\title{
Facile way of surface modification on hydrogel for drug delivery
}

\author{
Xin Wang, Pin Chen and Xiaohong $\mathrm{Hu}^{\mathrm{a}}$ \\ School of Material Engineering, Jinling Institute of Technology, Nanjing 211169, China
}

\begin{abstract}
Although hydrogel has some outstanding performance, traditional hydrogel still has some drawbacks in the application of drug delivery field. Herein, we introduced a simple and effective way to improve the performance of tradition hydrogel for drug delivery. $\beta$-cyclodextrin ( $\beta$-CD) functionized hydrogel was acquired through a two-step facile way, that is, alkaline hydrolysis and epichlorohydrin crosslinking. Though the reaction ratio of alkaline hydrolysis and the grafting ratio of $\beta-C D$ varied slightly according different detection methods, successful modification as well as proper properties including contact angle, equilibrium water content and surface morphology was still approved through the results of this work. The equilibrium amount of loading orfloxacin in hydrogels increased significantly with the increase of drug concentration and the equilibrium amount of loading orfloxacin in $\beta$-CD modified hydrogel was superior to that in traditional hydrogel with significant difference. The loading drug concentration as well as $\beta-\mathrm{CD}$ had effects on drug release behaviors.
\end{abstract}

Keywords: surface modification; hydrogel; drug delivery; $\beta$-cyclodextrin ( $\beta$-CD).

\section{Introduction}

Since hydrogel was first used as a soft contact lens for eyesight correction and cosmetology, the semi-solid material, which is composed of hydrophilic polymer network and is filled with enormous water in the polymer network, attracts intensive attention, especially in the field of tissue engineering and drug delivery[1-3]. Hydrogel is assumed to have good biocompatibility on account of aquatic environment, which brings about enormous research interest in recent years [4-6]. For this purpose, a growing number of investigations are focus on the evaluation of efficiency and properties of hydrogel including swelling property, biocompatibility, stimuli-responsive properties, and drug controlling behaviors [7-9]. It is reported that aquatic environment of hydrogel can protect cells, maintain the bioactivity of susceptible drugs, benefit the ions and molecules exchange between polymer network and outside environment $[1,2]$. These properties endow hydrogel an ideal drug carrier. As for drug carriers, drug encapsulated capacity and drug controlled-release properties are required for hydrogel. However, hydrophilic polymer network of traditional hydrogel had limited drug encapsulated capacity and drug controlled ability, which also had been discovered in our previous researches[10, 11].

Recently, some methods have been used to enhance interactions between drugs and hydrogel network including copolymerization with ionic monomers, introducing nanoparticles or colloids and using molecularly imprinted technology[8, 11, 12]. Although these systems improve the performance

a Corresponding author : hxh@jit.edu.cn 
of hydrogel as a drug carrier, they still cannot solve all problems of hydrogel for drug delivery. Moreover, some fabrications of these systems are complex to large-scale production. Even the drug encapsulated capacity and the drug controlled-release properties of hydrogel are improved at the expense of other performances such as elasticity, toughness, lubricity, transparency and the like[10, 11]. Therefore, the aim of this study is to provide a facile and effective method to improve the performance of tradition hydrogel for drug delivery.

Cyclic oligosaccharides (CDs) exert an intensive influence on the field of drug delivery, and are considered as an idealintermediate to hold drug molecules on account of host-guest interaction between hydrophobic cavity of CDs and drug molecules[13, 14]. As a member of cyclic oligosaccharides, $\beta-C D$ is easily obtained and modified. Meanwhile, with the advantage of maintaining the original properties of materials, surface modification is a simple and effective way to improve a certain performance of materials $[15,16]$. Further, with regard to polyester material, slight surface erosion through alkali and amine to endow material reactive groups such as $-\mathrm{NH}_{2}$ and $-\mathrm{COOH}$ is a proven and effective method for further surface modification. Since poly-hydroxyethyl methacrylate (pHEMA) is also a kind of polyester material, pHEMA traditional hydrogel was hydrolyzed in alkaline solution to produce - $\mathrm{COOH}$ groups in this work. Then $\beta$-CD was grafted onto pHEMA traditional hydrogel through epichlorohydrin crosslinking to improve drug encapsulated capacity and drug controlled ability of hydrogel.

\section{Experimental}

\subsection{Materials}

Hydroxyethyl methacrylate (HEMA) was obtained from Shanghai Jingchun Industries Co. Ltd, China, and distilled under vacuum before use. $\beta$-cyclodextrin, ammonium persulphate (APS) and N,N,N',N'-tetramethylethylenediamine (TEMED) were obtained from Shanghai Chemical Industries Co. Ltd (China). Orfloxacin was purchased from Zhengzhou Andrew Biological Engineering Co., LTD, China. All other reagents and solvents were of analytical grade and used as received.

\subsection{The surface modification}

pHEMA hydrogel was formed by previous method[17]. Brielfly, $500 \mu \mathrm{L}$ of $65 \%$ HEMA monomer solution, which contained $0.5 \%$ initiators of APS and TEMED (equal molar ratio), were injected into a circle model $\left(200 \mu \mathrm{m}\right.$ thickness), and then formed at $60{ }^{\circ} \mathrm{C}$ after $1 \mathrm{~h}$ later. Then hydrogel was immersed in $8 \mathrm{~mol} / \mathrm{L} \mathrm{NaOH}$ solution for $30 \mathrm{~min}$ at $70^{\circ} \mathrm{C}$, which was subsequently washed with water. Meanwhile, about $105 \mathrm{mg} \beta-\mathrm{CD}$ was dissolved in $10 \mathrm{~mL}$ water, into which $0.21 \mathrm{~mol}$ epichlorohydrin was dropped under stirring. After the above reaction lasted for $30 \mathrm{~min}$, a piece of hydrogel after alkaline hydrolysis was added for further $5 \mathrm{~h}$ reaction. Finally, hydrogels were washed with water.

Hydrogels were characterized by Fourier-transformed infrared spectroscopy (FTIR, Nicolet IS10), contact angle measurement system (Kruss, DSA100), element analysis (Eager 300). After freeze-dried, the hydrogels were observed by scanning electron microscopy (SEM, SU8100, Hitachi Japan). The dry hydrogels $\left(W_{0}\right)$ were submerged in water at $37{ }^{\circ} \mathrm{C}$ for $12 \mathrm{~h}$ and weighed $\left(W_{1}\right)$. The equilibrium swelling ratio of the hydrogels was defined as ESR $(\%)=\left(W_{1}-W_{0}\right) / W_{1} \times 100 \%$. The $\beta-\mathrm{CD}$ content was detected using 3-methylbenzoic acid (3-MBA) method [6]. Briefly, dried hydrogel were immersed in 3-MBA aqueous solution $(10 \mathrm{~mL}, 0.5 \mathrm{mg} / \mathrm{ml})$ in the dark. The 3-MBA concentration was spectrophotometrically determined (cary 50 ) at $281 \mathrm{~nm}$. The number of $\beta$-CD cavities was estimated from the amount of absorbed 3-MBA, which was calculated as the difference between the initial and the final amounts in the solution. 


\subsection{Drug loading and releasing}

Hydrogels were submerged in $3 \mathrm{ml}$ orfloxacin solution with different concentration to load drugs for $24 \mathrm{~h}$. The orfloxacin concentration was spectrophotometrically determined (cary 50) at $270 \mathrm{~nm}$ [6]. The loaded drugs in hydrogel were calculated by a difference of drug concentration before and after loading. The drug loaded hydrogels were submerged in $4 \mathrm{ml}$ PBS at $5 \mathrm{ml}$ centrifugal tube. At regular intervals, $1 \mathrm{ml}$ of released solution was moved from the centrifugal tube and $1 \mathrm{ml}$ of fresh PBS was added at the same time. The orfloxacin concentration at given time was also obtained using the above method, and the cumulative released amount was calculated according the orfloxacin concentration and volume.

\subsection{Statistical analysis}

Data were analyzed using the t-test for differences. Results are reported as means \pm standard deviation. The significant level was set at $\mathrm{p}<0.05$.

\subsection{Results and discussion}

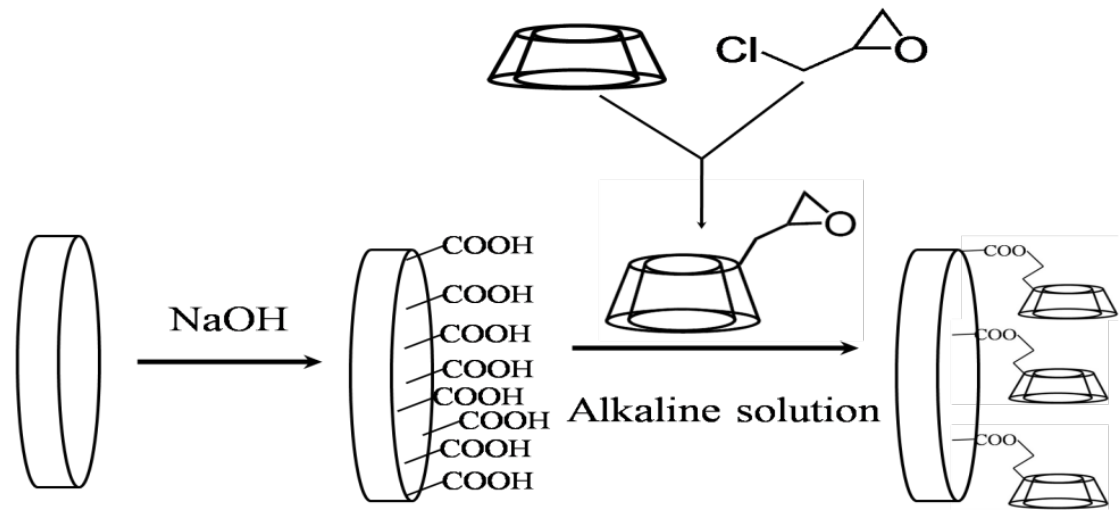

Figure 1. Schematic illustration to show hydrogel surface modification.

The surface modification was conducted through two procedures, the first of which introduced carboxy group on the surface of hydrogel through alkaline hydrolysis, the second of which grafted $\beta-\mathrm{CD}$ onto hydrogel surface through epichlorohydrin crosslinking at alkaline environment (Fig. 1a). The synthesis process was characterized by FTIR, which shown in Fig. 2. The peak of $1710 \mathrm{~cm}^{-1}$ belonging to ester groups and the peak of $1070 \mathrm{~cm}^{-1}$ belonging to hydroxyl groups emerged in hydrogel surface (pHEMA)[18], hydrogel after alkaline hydrolysis (pHEMA-COOH), and $\beta-\mathrm{CD}$ modified hydrogel (pHEMA-CD) without significant difference. However, since ester amount of each sample coming from pHEMA were defined, ester amount was utilized as a standard to normalize hydroxyl group of $1070 \mathrm{~cm}^{-1}$. The relative absorbance and relative area for hydroxyl group were specific in the Table 1. It is found that both relative absorbance and relative area for $\beta$-CD modified hydrogel (pHEMA-CD) were larger than that for pure pHEMA (pHEMA) and hydrogel after alkaline hydrolysis (pHEMA-COOH), which indicated that $\beta-\mathrm{CD}$ was successfully grafted onto hydrogel. Further, the reaction ratio was calculated according to element analysis, weight variation, and $\beta-\mathrm{CD}$ content, which were specific in Table 1 . Though the reaction ratios differ from different methods, the alkaline hydrolysis ratio was around $10 \%$, and $\beta$-CD grafting ratio was lower than $1 \%$. Moreover, surface modification induced a slight variation of hydrogel properties such as hydrophilicity coming from contact angle and equilibrium water content (Table 1), which demonstrated that surface modification had slight effects on the properties of hydrogel. Nevertheless, the morphology of hydrogel (Fig. 3 varied after surface modification. The hydrogel (pHEMA) showed a flat and smooth surface (Fig. 3a), while the alkaline hydrolysis (pHEMA-COOH) brought about coarseness and even 
some defects on the hydrogel surface (Fig. 3b), and the surface of modified hydrogel (pHEMA-CD) were further deteriorated (Fig. 3c).

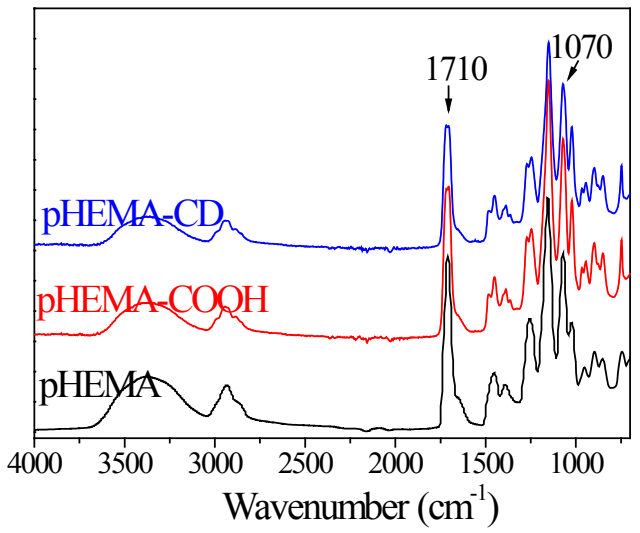

Figure 2. FTIR spectra of hydrogel surface before and after modification.

Table 1. The grafting reaction condition, grafting ratio, XPS and FTIR characterizations of pHEMA hydrogel

\begin{tabular}{|c|c|c|c|c|c|c|c|}
\hline \multirow{2}{*}{ Entry } & \multicolumn{3}{|c|}{$\begin{array}{l}\text { Reaction ratio though different } \\
\text { methods }(\%)\end{array}$} & \multicolumn{2}{|c|}{$\begin{array}{c}\text { FTIR } \\
\text { characterizations }\end{array}$} & \multirow{2}{*}{$\begin{array}{c}\text { Contact angle } \\
\left({ }^{\circ}\right)\end{array}$} & \multirow{2}{*}{$\begin{array}{l}\text { Equilibriu } \\
\text { m water } \\
\text { content (\%) }\end{array}$} \\
\hline & $\begin{array}{c}\text { weight } \\
\text { variation }\end{array}$ & $\begin{array}{l}\text { element } \\
\text { analysis }\end{array}$ & $\begin{array}{c}\beta-C D \\
\text { detection }\end{array}$ & $\begin{array}{l}\mathbf{A}_{1070} / \mathbf{A}_{17} \\
10\end{array}$ & $\begin{array}{c}\mathbf{I}_{1070} / \mathbf{I}_{171} \\
0\end{array}$ & & \\
\hline pHEMA & I & / & l & 0.78 & 1.02 & $68.3 \pm 1.9$ & $46.5 \pm 0.8$ \\
\hline $\begin{array}{c}\text { pHEMA-COO } \\
\text { H }\end{array}$ & $13.0 \pm 3.2$ & 9.6 & / & 0.72 & 0.99 & $65.6 \pm 2.0$ & $47.9 \pm 1.1$ \\
\hline pHEMA-CD & $\begin{array}{c}0.58 \pm \\
0.16\end{array}$ & I & $\begin{array}{c}0.40 \pm \\
0.11 \\
\end{array}$ & 1.05 & 1.33 & $74.1 \pm 1.4$ & $48.2 \pm 0.9$ \\
\hline
\end{tabular}

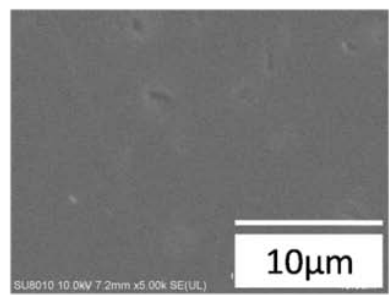

a

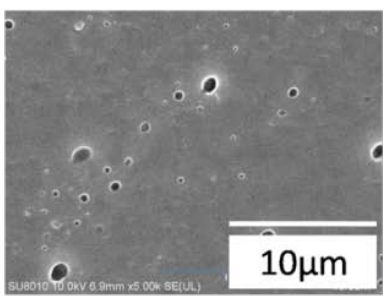

b

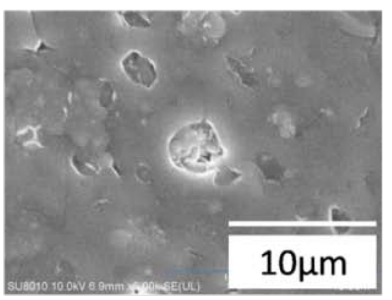

C

Figure 3. SEM micrograph of pHEMA(a), pHEMA-COOH(b) and pHEMA-CD(c)

In order to examine drug loading and releasing capacity of hydrogel, orfloxacin was loaded into hydrogels through a soaking method. It was found that hydrogels could be loaded with orfloxacin to equilibrium after they were immersed in drug solutions for $24 \mathrm{~h}$, regardless of drug concentration. The equilibrium amount of loading orfloxacin in hydrogels increased significantly with the increase of drug concentration, as shown in Fig. 4. Not surprisingly, higher drug concentration resulted in more drug molecules being spread into hydrogel network (Fig. 4), since the diffusion is a main driving force to load orfloxacin through a soaking method[7]. With respect to drug encapsulated capacity for different hydrogels, the equilibrium amount of orfloxacin loading in $\beta$-CD modified hydrogel (pHEMA-CD) was superior to that in traditional hydrogel (pHEMA) with significant difference (Fig. 
5). This may be attributed to the formation of inclusion complexes between hydrophobic $\beta$-CD groups in hydrogel and orfloxacin molecules, which enhance the affinity of between hydrogel network and orfloxacin molecules. The release behaviors of orfloxacin from hydrogels with different drug concentration were shown in Fig. 5. At the beginning $2 \mathrm{~h}, 90 \%$ orfloxacin was released fast from presoaked traditional hydrogel (pHEMA) when the loading drug concentration was at $0.15 \%$ (Fig. 5a). Then, the remained orfloxacin was gradually released from the hydrogel in subsequent $22 \mathrm{~h}$. By comparing, the release speed of orfloxacin from presoaked $\beta-\mathrm{CD}$ modified hydrogel (pHEMA-CD) was slower than that from presoaked traditional hydrogel after first burst release in half an hour. Similarly, the $10 \%$ remained orfloxacin was gradually released from the $\beta-C D$ modified hydrogel (pHEMA-CD) after the $4 \mathrm{~h}$ beginning fast release. The drug release rate is controlled by diffusion of free drug from the aqueousphase of hydrogel and the dissociation of absorbed drug in hydrogel network. For traditional hydrogel, the beginning rapid drug release rate came from diffusion of free drug, and the slow orfloxacin release rate only came from the dissociation of absorbed drug by polymer backbone[7]. While for $\beta$-CD modified hydrogel (pHEMA-CD), $\beta$-CD/drug inclusion complexes was also a driving force to control drug release besides the diffusion and dissociation force. When the loading drug concentration was at $0.25 \%$ (Fig. 4b), the drug release behaviors from either presoaked traditional hydrogel (pHEMA) or presoaked $\beta$-CD modified hydrogel (pHEMA-CD) exhibited similar trends with low loading drug concentration. However, the loading drug concentration was at $0.68 \%$, the influence of $\beta-\mathrm{CD} / \mathrm{drug}$ inclusion complexes on controlling ability of drug release was no longer obvious (Fig. 4d). The result might be ascribed to a large-scale free drug molecules in $\beta-C D$ modified hydrogel network, which reduce the influence of the driving force coming from $\beta-\mathrm{CD} / \mathrm{drug}$ inclusion complexes. Therefore, it is inferred from these results that $\beta$-CD on hydrogel surface improved the drug encapsulated capacity not only through accommodating drug molecules in cavity of $\beta-\mathrm{CD}$, but also benefiting the free drug absorption.

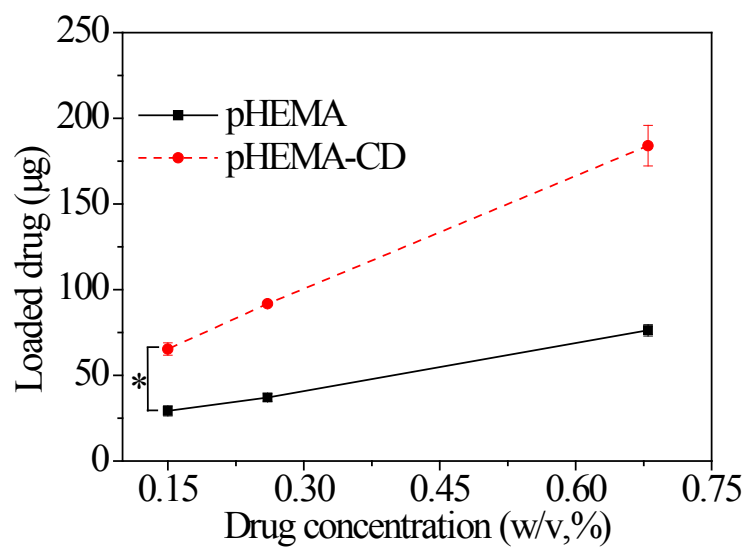

Figure 4. The loaded drug amount in different hydrogels as a function of drug concentration. 


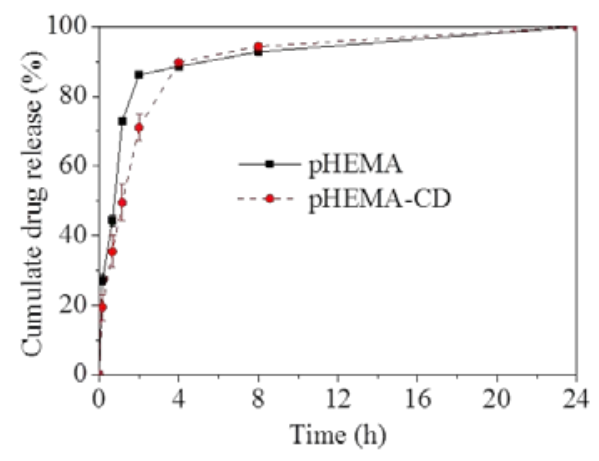

a
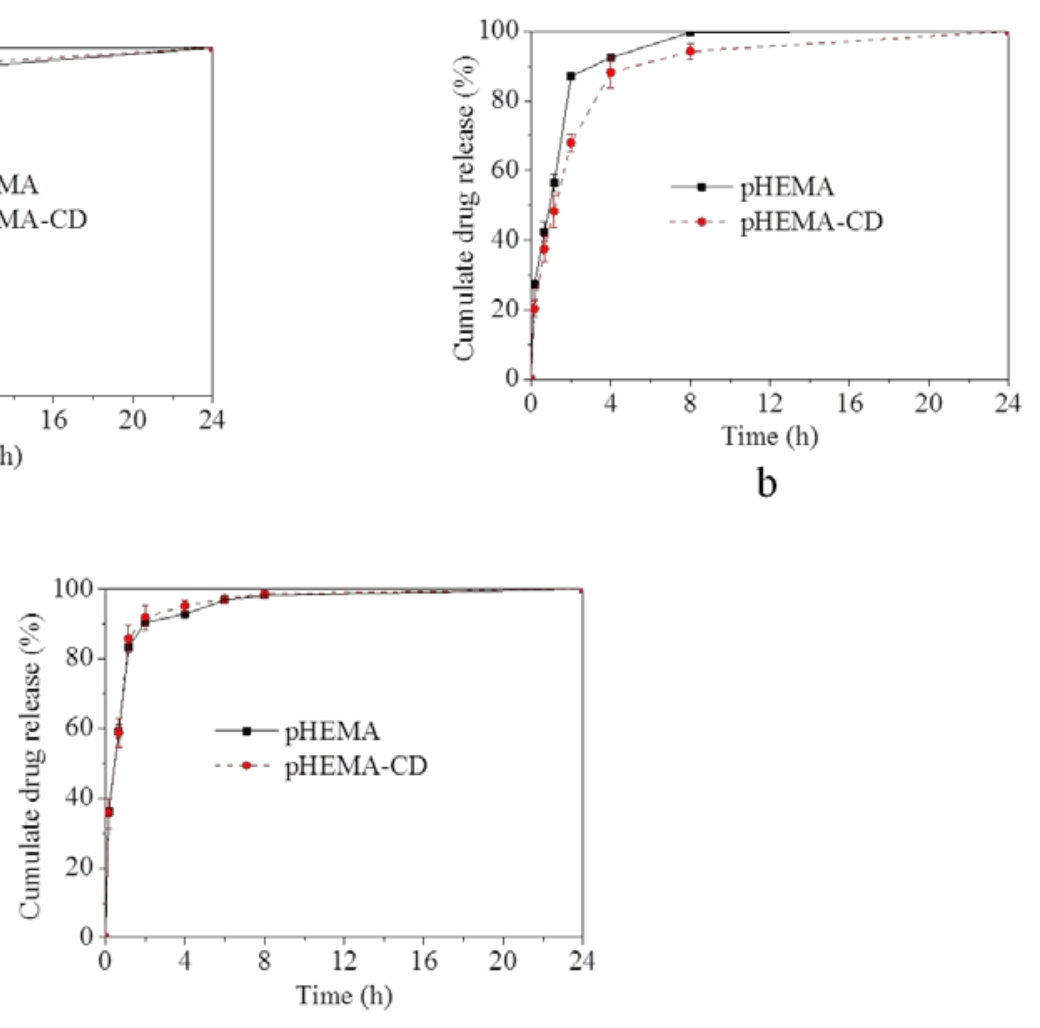

$\mathrm{C}$

Figure 5. The cumulate orfloxacin release behaviors of hydrogels when the drug concentration was at $0.15 \%$ (a), $0.25 \%(\mathrm{~b})$ and $0.68 \%(\mathrm{c})$.

\section{Conclusions}

$\beta$-cyclodextrin ( $\beta$-CD) functionized hydrogel was successfully obtained through a two-step facile way, that is, alkaline hydrolysis and epichlorohydrin crosslinking. The reaction ratio of alkaline hydrolysis was around $10 \%$, and the grafting ratio of $\beta-\mathrm{CD}$ was below $1 \%$. Though the reaction ratio of alkaline hydrolysis and the grafting ratio of $\beta-C D$ varied slightly according different detection methods, successful modification as well as the proper properties were still approved through the results of this work. Surface modification had slight effects on the contact angle and equilibrium water content. However, surface morphology was influenced by alkaline hydrolysis and epichlorohydrin crosslinking. The equilibrium amount of loading orfloxacin in hydrogels increased significantly with the increase of drug concentration, and the equilibrium amount of loading orfloxacin in $\beta$-CD modified hydrogel was superior to that in traditional hydrogel with significant difference. The loading drug concentration as well as $\beta$-CD had effects on drug release behaviors. $\beta-C D$ in hydrogel showed some controlled abilities on drug release behavior when the loading drug concentration was low. However, the effects of $\beta$-CD on drug release behavior decreased with the increase of loading drug concentration.

\section{Acknowledgments}

This study is financially supported by Qing Lan Project, and Research Fund of Jinling Institute of Technology in China (JIT40410425). 


\section{References}

1. X. H. Hu, D. Li, F. Zhou, C. Y. Gao, Acta Biomater., 7: 1618 (2011)

2. H. P. Tan, C. Xiao, J. Sun, D. S. Xiong, X. H. Hu, Chem. Commun. 48: 10289 (2012)

3. X. H. Hu, H. Q. Wang, X. L. Yang, G. J. Zhang, G. Y. Wang, X. J. Zhang, Int. J. Poly. Sci. 814163 (2011)

4. E. Barbu, L. Verestiuc, T. G. Nevella, J. Tsibouklis, J. Mater, Chem, 16: 3439(2006)

5. J.K. Cho, J.W. Park, S.C. Song, J. Pharm. Sci. 101: 2382(2012)

6. J.F. dos Santos, C. Alvarez-Lorenzo, M. Silva, L. Balsa, J. Couceiro, J.J. Torres-Labandeira, A. Concheiro, Biomaterials, 30: 1348( 2009)

7. M. Gou, X. Li, M. Dai, C. Gong, X. Wang, Y. Xie, H. Deng, L. Chen, X. Zhao, Z. Qian, Y. Wei Int. J. Pharm, 359: 228(2008)

8. Y. Kapoor, J.C. Thomas, G. Tan, V.T. John, A. Chauhan, Biomaterials, 30: 867 (2009)

9. J. Xu, X. Li, F. Sun, Acta Biomater. 6: 486(2010)

10. X. H. Hu, H. P. Tan, D. Li, X. H. Ma, J. Macromol. Sci, Part A, 50:983(2013)

11. X. H. Hu, H. P. Tan, D. Li, Y. Zhang, C. Pan, J. Macromol. Sci., Part A, 50: 1201(2013)

12. C. Alvarez-Lorenzo, F. Yanez, R. Barreiro-Iglesias, A. Concheiro, J. Control. Release., 113: 236( 2006)

13. T.R. Thatiparti, A.J. Shoffstall, H.A. von Recum, Biomaterials, 31: 2335(2010)

14. T.R. Thatiparti, H. A. von Recum, Macromol. Biosci., 10:82(2010)

15. X. H. Hu, G.J. Zhang, H.P. Tan, D. Li, X. X. Chen, Y.S. Zhang, Materi. Tech., 29: 144(2014)

16. X.H. Hu, H. P. Tan, D. Li, M.Y. Gu, Mater. Tech., 29: 8(2014)

17. X.H. Hu, D. Li, Mater. Tech., 28: 192(2013)

18. T. Perova, J. Vij, H. Xu, Coll. Poly. Sci., 275: 323 (1997) 